\title{
Unilateral pulmonary hypoplasia in an adult patient
}

\author{
Dimitrios Papadopoulos', Panagiotis Misthos², Maria Chorti³, Vlasios Skopas', \\ Alexandra Nakou1, Napoleon Karagianidis', Achilleas Lioulias², Vasiliki Filaditaki1
}

$12^{\text {nd }}$ Pulmonary Department, "Sismanoglio" General Hospital of Attica, Marousi

2 Thoracic Surgery Department, "Sismanoglio" General Hospital of Attica, Marousi

3 Pathology Laboratory, "Sismanoglio" General Hospital of Attica, Marousi, Greece

\begin{abstract}
Pulmonary hypoplasia (PH) is a developmental anomaly of the lung parenchyma, characterized by a decrease in the number and size of airways, alveoli and vessels. We present a case of a 31-year-old patient with a history of chronic productive cough and frequent respiratory infections, who was referred for investigation of abnormal chest x-ray. The combination of chest computed tomography (CT) and bronchoscopy set the diagnosis of left pulmonary hypoplasia and the patient was treated surgically with a left pneumonectomy. PH is usually diagnosed immediately after birth, causing severe respiratory failure with high mortality. The less severe, unilateral forms can possibly survive by causing compensatory hyperinflation of the other lung and remain undiagnosed until adulthood, presenting either asymptomatic or with symptoms of chronic bronchitis and recurrent respiratory infections. Chest CT is considered the imaging technique of choice for the diagnosis and for the differential diagnosis from other congenital or acquired conditions. The treatment is usually conservative, although surgical resection is indicated in cases of severe cystic changes and intense symptomatology.
\end{abstract}

Corresponding author: Dimitrios Papadopoulos, "Sismanoglio" General Hospital of Attica, Sismanogliou 1, 15126 Marousi, Greece.

Tel. +30.213.2058422 - Fax: +30.2132.058099.

E-mail: d.g.papadopoulos@gmail.com

Key words: Pulmonary hypoplasia; computed tomography; pneumonectomy

Contributions: DP, PM, MC, VS, conception, design, drafting, revising, final approval; AN, NK, AL, VF, conception, revising, final approval.

Conflict of interest: The authors declare no financial support or conflict of interest.

Received for publication: 17 April 2017

Accepted for publication: 10 December 2017

(C) Copyright D. Papadopoulos et al., 2018

Tipografia PI-ME Editrice, Italy

Monaldi Archives for Chest Disease 2018; 88:829

doi: 10.4081/monaldi.2018.829

This article is distributed under the terms of the Creative Commons Attribution Noncommercial License (by-nc 4.0) which permits any noncommercial use, distribution, and reproduction in any medium, provided the original author(s) and source are credited.

\section{Introduction}

Pulmonary hypoplasia (PH) constitutes a part of the spectrum of developmental anomalies that cause incomplete development of lung tissue and is characterized by a decrease in the number of lung cells, airways and alveoli, finally leading to a reduction in lung size and weight. It can be unilateral or bilateral and it usually leads to severe respiratory distress immediately after birth, while it represents a common cause of perinatal mortality [1].

The less severe forms of PH can survive and remain undiagnosed since adulthood, when they present either as an accidental imaging finding in asymptomatic patients or within investigation of recurrent lower respiratory tract infections (LRTI) and respiratory failure. Chest computed tomography (CT) is the diagnostic tool of choice [2]. A 31-year-old patient case with unilateral $\mathrm{PH}$ and history of frequent respiratory infections since childhood is presented.

\section{Case Report}

A 31-year-old, non-smoker, Albanian woman was referred to our hospital in July 2016 by a private doctor because of an abnormal chest $\mathrm{x}$ ray (CXR), performed in the context of an acute LRTI, for which she received levofloxacin for 10 days. In her medical history, she reported chronic productive cough and frequent episodes of LRTI since childhood, which were treated with antibiotics. She also mentioned a known abnormality on CXR that was attributed to repeating infections and was not investigated any further.

Upon admission, she was afebrile, hemodynamically stable, with normal respiratory rate and oxyhemoglobin saturation. Physical examination revealed reduced breath sounds in the left middle and lower lung field with dullness to percussion and decreased vocal fremitus. The laboratory tests showed an increase in inflammatory markers and CXR depicted opacity of the left hemithorax with ipsilateral mediastinal shift and increase in the volume of the right lung (Figure 1). Her tuberculin skin test was negative $(0 \mathrm{~mm})$. The patient was treated empirically with a combination of ampicillin-sulbactam and clindamycin. Chest CT was performed with findings of hypoplastic and contracted left lung with cystic changes of the airways, mediastinal shift to the left and compensatory emphysema of the right lung with herniation to the opposite side (Figure 2). No coexistent congenital skeletal, diaphragmatic, cardiac or kidney abnormality was identified. Spirometry revealed mild obstructive disorder (FVC 84\%, FEV1 74\%, FEV1/FVC 77\%, MMEF 55\%). It was followed by bronchoscopy wherein uniform narrowing of the left bronchial tree was observed with chronic inflammatory lesions, while the right bronchial tree had normal appearance. Cultivation of the bronchial secretions isolated Haemophilus influenza, while it was negative for fungi, Nocardia and mycobacteria. We recom- 
mended continuation of antibiotic therapy with amoxicillin-clavulanate for two weeks and planning for surgical resection in order to relieve the chronic symptomatology.

After 2 months, the patient was readmitted for elective surgery. A left posterolateral thoracotomy was performed. The pleural cavity was filled with the pericardial sac. The left lung was a small one, occupying the posterior costophrenic recess. The contralateral right lung filled the upper half of the left pleural space. The left hypoplastic lung was a miniature with normal bronchial and vascular anatomy, while no aberrant connection with the systematic circulation was encountered. A left pneumonectomy was done. The pathological specimen weighed $136 \mathrm{~g}$, had dimensions of $15.5 \times 9 \times 3.7 \mathrm{~cm}$ and a macroscopic tubular look of the parenchyma alternating with islets of fibrotic or adipose tissue. Microscopic examination revealed a complete disruption of the architecture in the presence of confluent stretched

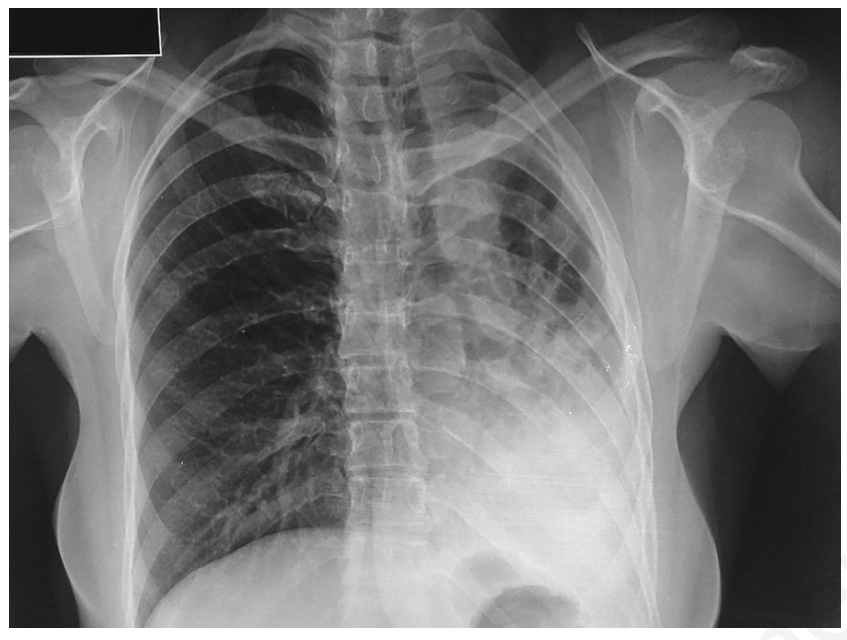

Figure 1. Postero-anterior chest radiograph showing opacity and volume reduction of the left hemithorax, with presence of cysticlike formations instead of normal parenchyma and mediastinal shift to the left. bronchial branches with or without cartilage wall and chronic inflammation lesions alternating with equally confluent vascular spaces with unregulated distribution relatively to the bifurcation of the bronchi, as well as an increase of the interstitial layer in the form of fibrous connective or adipose tissue (Figure 3). The postoperative course was uneventful and the patient was discharged on the $6^{\text {th }}$ postoperative day.

The imaging and histological examination was considered compatible with the diagnosis of PH and patient was recommended regular monitoring and prophylactic vaccination against influenza and Streptococcus pneumoniae.

\section{Discussion}

In 1912, Schneider categorized the developmental abnormalities of the lung parenchyma into three degrees, as follows: i) complete absence of parenchyma, vessels and bronchi (agenesis); ii) presence of a rudimentary main bronchus that ends in a blind pouch without development of parenchyma or vasculature (aplasia); and iii) presence of hypoplastic vessels and bronchi of varying degrees with corresponding variable reduction of lung tissue (hypoplasia) [3]. The severity of $\mathrm{PH}$ depends on the timing of insult in association with the embryonic stage of development of the lung tissue. Impairment before the $16^{\text {th }}$ gestational week results in both reduced bronchiolar branching and delayed acinar development, while at later stages it influences only acinar development [4]. The incidence of $\mathrm{pH}$ in the general population ranges from 9 to 11 per 10000 live births, but not including the least severe cases without symptoms at birth. Perinatal mortality is reported high (55-100\%) and its prevalence in neonatal autopsies is in the order of $8-22 \%$, while more than $85 \%$ of them coexist with other related developmental abnormalities [1].

Physical factors affecting the normal development of the fetal lung is the intrathoracic capacity, the volume of amniotic fluid, the volume and pressure of pulmonary fluid, respiratory movements and blood supply, while transcription and growth factors are involved in the regulation of the process [5]. So, the etiology of PH may involve space-occupying lesions of the chest; skeletal malformations or large defects in the ab-

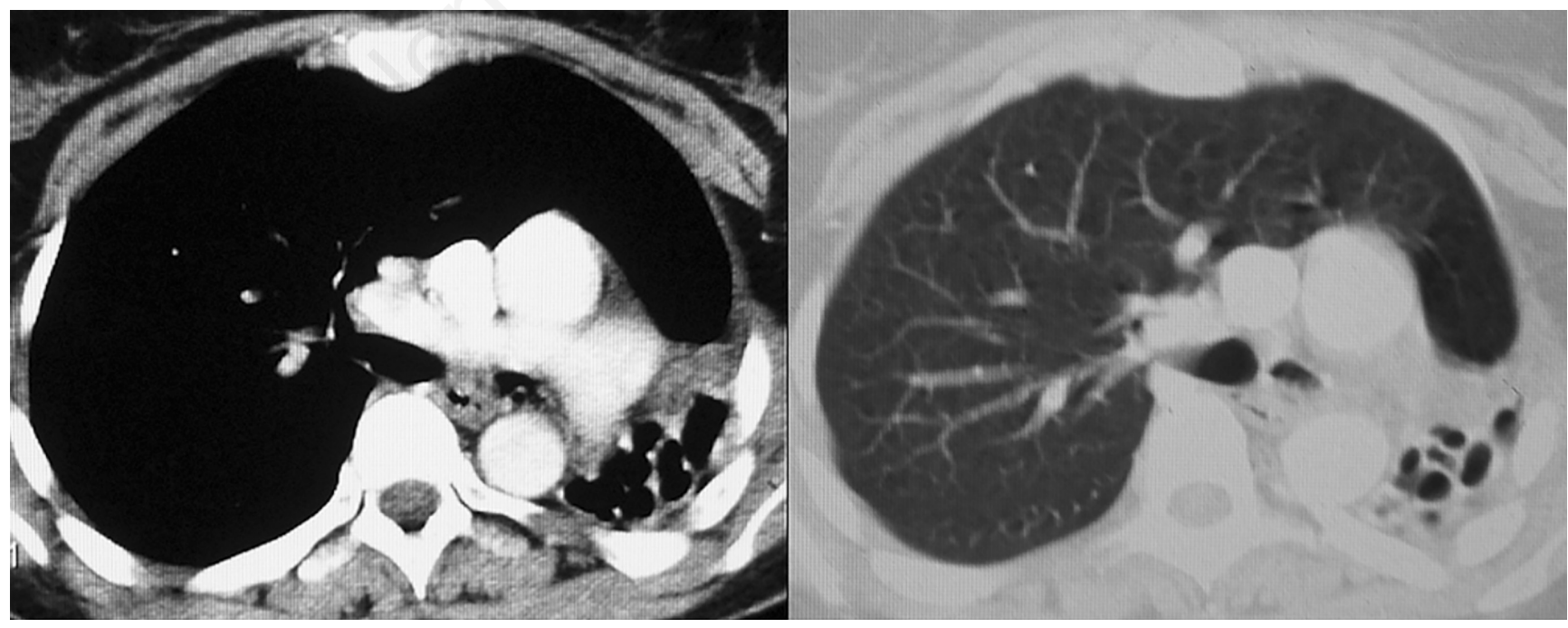

Figure 2. Chest CT scan showing hypoplasia and cystic degeneration of the left lung, hyperinflation of the right lung with herniating on the opposite side and mediastinal shift to the left. A decrease in size of the left main bronchus and the main branch of the left pulmonary artery is observed, without distinction of further bifurcations. 
dominal wall that limit the capacity of the rib cage; abnormalities of the urinary tract or non-renal causes of oligohydramnios that cause loss of lung fluid; neuromuscular or central nervous system abnormalities disrupting fetal breathing movements; cardiac lesions affecting the pulmonary blood supply; and chromosomal abnormalities with a genetic background [6]. In rare cases, no apparent cause is recognized, so they are referred to as primary (idiopathic) PH. In some of these a familial occurrence has been recognized and the hypothesis of a lack of transcription or growth factors regulating growth of normal lung epithelium, which are possibly inherited in an autosomal recessive mode, has been proposed [17].

Clinical manifestations in neonates range from severe respiratory failure requiring prolonged mechanical ventilation and leading to early death (fatal form), to respiratory insufficiency associated with pulmonary hypertension, pulmonary hemorrhage, bronchopulmonary dysplasia or subacute lung disease, to mild or transient symptomatology (non-fatal form) [6]. In imaging, the affected hemithorax is asymmetrically small with hemidiaphragm elevation and variable amounts of mediastinal shift. Hyperinflation of the contralateral lung may be present and can herniate towards the opposite side [8]. Histological findings are nonspecific and the diagnosis is confirmed by autopsy when the ratio of lung weight to body weight is less than 0.012 and/or radial alveolar count is less than $4.1 \%$ [9].

The diagnosis of $\mathrm{PH}$ in adults is rare. Comet et al. [2] identified in 1998 only 48 cases published in the literature, but considered possible the presence of cases, whose imaging findings were incorrectly attributed to old tubercular infections, often unproven. The left lung is most commonly affected and coexisting conditions, such as hiatal hernia, kidney or skeletal abnormalities, may be identified. Clinical manifestations are variable and non-specific, with patients presenting either asymptomatic or with symptoms of chronic bronchitis or advanced respiratory failure, depending on the time of diagnosis. Pulmonary function tests usually demonstrate a restrictive disorder of variable severity, but in some cases, repeated LRTI, age and hyperinflation of the healthy lung can add an obstructive component. Suspicion of the disease is based on a CXR showing opacity and decrease in the width of the intercostal spaces of the affected hemithorax, with mediastinal shift and herniation of the healthy lung ipsilaterally. Chest CT has re-

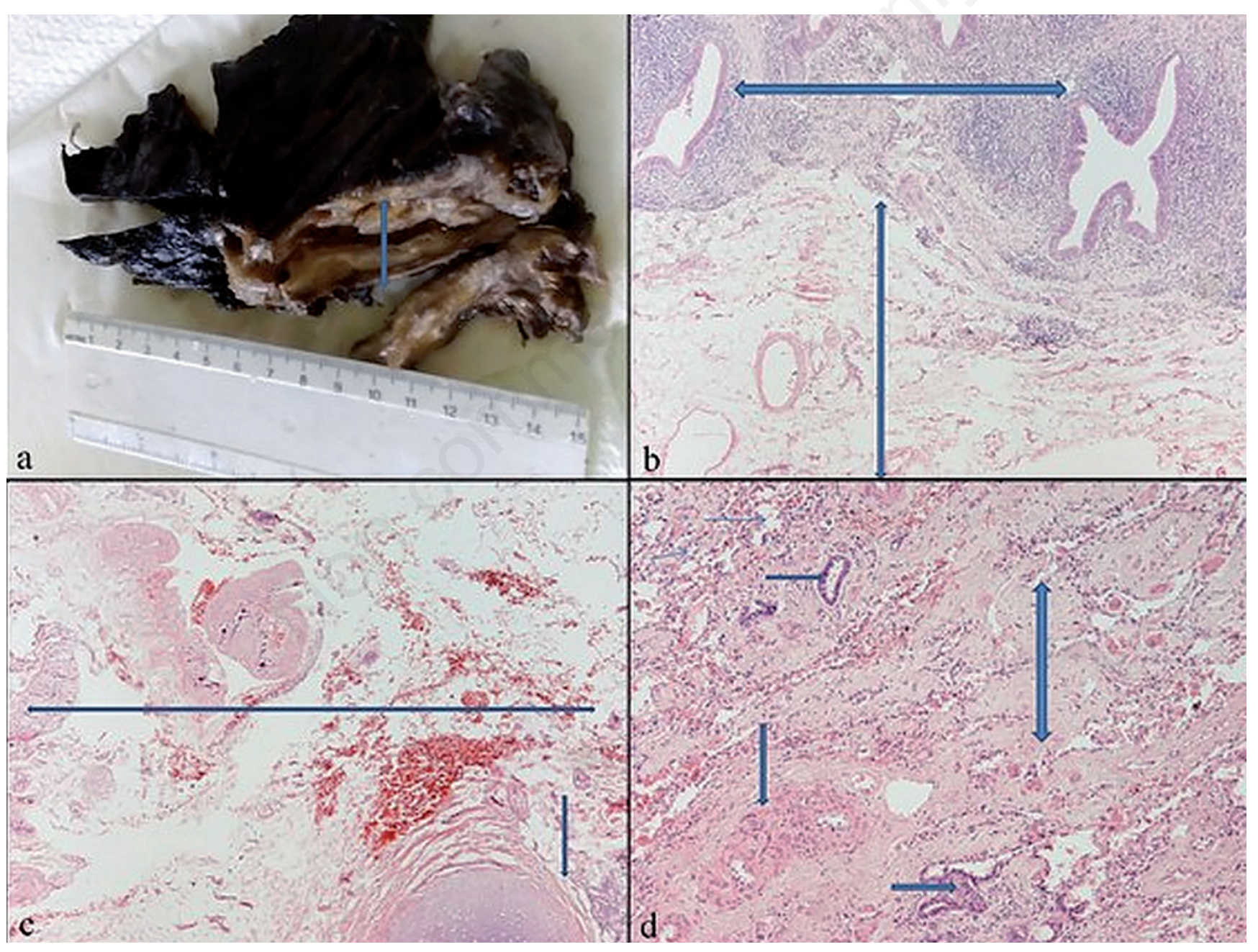

Figure 3. a) Contracted pneumonectomy specimen with dimensions of $15.5 \times 9 \times 3.7 \mathrm{~cm}$ and tubular fibro-elastic sectional area (vertical arrow), without the "texture" of pulmonary parenchyma (pigmented pleural surface with indian ink). b) Abnormal architecture of the bronchial tree: confluent bronchial branches (horizontal double arrow) with adjacent vascularized adipose tissue instead of pulmonary parenchyma (vertical arrow). c) Hyaline cartilage (vertical arrow) and vascularized, hemorrhagically infiltrated fibro-fatty tissue without lung parenchyma (horizontal arrow). d) Fully fibrotic lung parenchyma (vertical double arrow) with minimum atrophic bronchiolar branches (right arrow), a limited number of "alveolar" areas (thin arrows) and vessels (vertical arrow) (hematoxylin-eosin stain x40). 
placed bronchography and pulmonary angiography in the study of the deformed and hypoplastic bronchial and vascular tree and is the diagnostic test of choice, while allowing the recognition of coexisting anomalies [2].

The differential diagnosis will involve the following situations: i) secondary (acquired) pneumopathy with non-congenital bronchiectasis (pseudo-hypoplasia), as after surgical resection or tuberculosis; ii) cystic adenomatoid malformation, characterized by the presence of multiple cystic formations expanding and displacing the normal parenchyma and shifting the mediastinum to the opposite side; iii) bronchopulmonary sequestration with imaging characteristics of a pulmonary mass without normal bronchial communication; and iv) congenital lobar emphysema, which mimics the compensatory hyperinflation of the contralateral lung in $\mathrm{PH}$, with the difference that the architecture of bronchial and vascular bed of the smaller lung is maintained normal [10].

The treatment in adults is usually conservative and consists of regular monitoring, treatment of infections, symptomatic relief with bronchodilators or expectorants and prophylactic vaccination. In some patients, the combination of abnormal angulation of the airways, defective mucociliary clearance and pooling of secretions in blind hypoplastic ends leads to recurrent infections and cystic degeneration of the airways, so there is an indication for surgical removal of the affected lung [11].

\section{Conclusions}

In conclusion, $\mathrm{PH}$ refers to a rare diagnosis in adults and may be asymptomatic or associated with repeated LRTI. Chest CT must necessarily be carried out in each case of a contracted lung on plain radiography in a patient with no known history of tubercular infection. Surgical resection of the affected lung is indicated in patients with pronounced symptoms and without severe pulmonary function impairment.

\section{References}

1. Laudy JA, Wladimiroff JW. The fetal lung. 2: pulmonary hypoplasia. Ultrasound Obstet Gynecol 2000;16:482-94.

2. Comet R, Mirapeix RM, Marín A, et al. Pulmonary hypoplasia in adults: embryology, clinical presentation and diagnostic methods. Our experience and review of the literature. Arch Bronconeumol 1998;34:48-51.

3. Schneider P. Die Missbildungen der Atmungsorgane. In: Schwalbe E, editor. Die Morphologie der Missbildungen des Menschen und der Tiere, vo1. 3: Die Einzelmissbildungen, Abt. 2: Missbildungen der einzelnen Organe und Organsysteme. Jena: Gustav Fischer; 1912. pp.763-857.

4. Nakamura Y, Harada K, Yamamoto I, et al. Human pulmonary hypoplasia. Statistical, morphological, morphometric, and biochemical study. Arch Pathol Lab Med 1992;116:635-42.

5. Laudy JA, Wladimiroff JW. The fetal lung. 1: developmental aspects. Ultrasound Obstet Gynecol 2000;16:284-90.

6. Sherer DM, Davis JM, Woods JR Jr. Pulmonary hypoplasia: a review. Obstet Gynecol Surv 1990;45:792-803.

7. Moerman P, Vanhole C, Devlieger H, Fryns JP. Severe primary pulmonary hypoplasia ("acinar dysplasia") in sibs: a genetically determined mesodermal defect? J Med Genet 1998;35:964-5.

8. Thacker PG, Schooler GR, Caplan MJ, Lee EY. Developmental lung malformations in children: recent advances in imaging techniques, classification system, and imaging findings. J Thorac Imaging 2015;30:29-43.

9. Askenazi SS, Perlman M. Pulmonary hypoplasia: lung weight and radial alveolar count as criteria of diagnosis. Arch Dis Child 1979;54:614-8.

10. Georgescu A, Nuta C, Bondari S. 3D imaging in unilateral primary pulmonary hypoplasia in an adult: a case report. Case Rep Radiol 2011;2011:659586.

11. Thomas RJ, Lathif HC, Sen S, et al. Varied presentations of unilateral lung hypoplasia and agenesis: a report of four cases. Pediatr Surg Int 1998;14:94-5. 